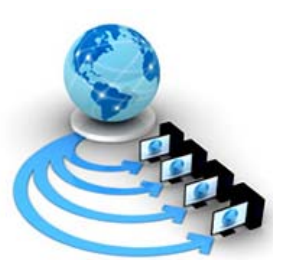

\title{
PREDICTING BREAST CANCER TUMOR BY MEANS OF AMMOGRAPHIC IMAGE PROCESSING
}

\author{
Nisha Dutta \\ CT Institute of Engineering Management and Technology, \\ Jalandhar, Punjab, India \\ Anurag Sharma \\ CT Institute of Technology and Research, \\ Jalandhar, Punjab, India
}

\author{
Harjeet Singh \\ CT Institute of Engineering Management and Technology, \\ Jalandhar, Punjab, India
}

\begin{abstract}
Breast cancer is a disease that accounts for a disturbingly large number of deaths in females each year. Although mammographic screening is the most effective method currently available for the early detection of breast cancer, it is far from being an infallible procedure. Mammographic reading is error prone, partly because of the complexity of the task and partly because of the variability in human performance. Computers offer high reproducibility, and when used as an adjunct by the radiologist, may improve diagnostic accuracy and thus the mammographic screening process. The goal of this research was to create using Mammogram Image Processing interface that support the complex segmentation, feature extraction, and classification algorithms.
\end{abstract}

Keywords: Malignant, Benign, Mammogram Image Processing.

\section{INTRODUCTION}

The most widespread disease today is Breast Cancer and is second biggest cause of deaths among women annually [6]. At present there is no effective way to prevent Breast Cancer because the main reason behind the disease is unknown. It is characterized by abnormal multiplication of cell in the human body. As it is diagnosed very late, this often causes very expensive treatments and may lead to serious problems. The widespread technique used for detecting breast cancer is Mammography, but it is very difficult to differentiate between breast tumors due to structural similarities between them [5]. So early detection of breast cancer helps in full recovery of the disease. Computer aided diagnosis or detection (CAD) system which uses computer technologies to detect abnormalities such as calcification, masses can play a key role in early detection of breast cancer and helpful in reducing death rate among women. Breast Cancer Tumors are of two types namely as Malignant Breast Tumor and Benign Breast Tumor [13].

Benign Tumors are non-cancerous cells which do not spread in the whole body but present in particular part of the body. When a tumor is diagnosed as benign, doctors will usually leave it alone rather than remove it. Even though these tumors are not generally aggressive toward surrounding tissue, occasionally they may continue to grow, pressing on organs and causing pain or other problems. In these situations, the tumor is removed allowing pain or complications to subside [8]. Malignant tumors are cancerous and aggressive because they invade and damage surrounding tissue. The main signs and symptoms of malignant tumor are change in skin color appears "orange pseudo" and breast lump become red and fixed. When a tumor is suspected to be malignant, the doctor will perform a biopsy to determine the severity or aggressiveness of the tumor. Malignant tumors spread from one part of body to other parts of the body and it are very difficult to remove [3,7]. There are various methods or techniques used for diagnosing breast cancer such as Mammography, Biopsy, Magnetic Resonance Imaging and Fine Needle Aspiration [9].

Mammograms are special type of X-ray imaging techniques to scrutinize breast cancer, and is presently the utmost operative scheme for the recognition of breast tumor. Mammograms propositions high-quality images at a low-slung radioactivity amount, and is presently the only extensively acknowledged imaging technique practiced for monotonous breast tumor identification [10]. Existing guiding principle of the American Cancer Society (ACS) endorse that women aged 40-49 years have a routine mammogram every single one to two years instigating at the age of 40 [2]. Mainly Mammograms are having two major technics, that are film mammography and digital mammography. In film mammograms, the mammogram is shaped directly on film, while digital mammograms are formed by capturing an electronic image of the breast and stores it directly on a computer. In primary type of Computer aided systems, the films are perused, digitized, and kept in the PC for additional investigation [11]. The secondary category of mammograms are customized to digital mammograms (FFDM) technology, which is predictable to deliver a better SNR with a better recognition quantum competence, broader dynamic assortment, and a advanced contrast compassion than digitized film mammography [4,5]. In this paper, to classify Breast Cancer Tumors (Malignant Tumors or Benign Tumors) using Mammogram imaging firstly image mammogram feature extraction is required, for which firstly mammogram data is acquired then next stage is image segmentation in which I have implemented the threshold technique using which the area of concern is extracted from the entire mammogram. One the entire image is segmented and tumor area is extracted then the second step is to extract the statistical features from the mammogram using various mathematical relationships implemented on mammogram using MATLAB m-file coding. Once the features are extracted we can observe the statically result to 
conclude the perimetric values for breast cancer tumor classifier.

\section{SYSTEM SETUP}

The images of mammogram that has been used are obtained from Digital Database for Screening Mammography (DDSM) for classification of breast masses. DDSM is a resource for use by mammographic analysis research communities and it is maintained by university of South Florida for the purpose of keeping it available on the web [1,15]. The experiment is conducted by using different images of the mammogram. For classification of masses, an experiment was conducted on 20 images of mammogram out of which some are malignant masses and some are benign masses. A sequence of steps which are to be followed before extracting features from the Mammogram for efficient and accurate calculation of statistical features from statistical analysis of mammogram images is performed in three stages as shown in figure 1.

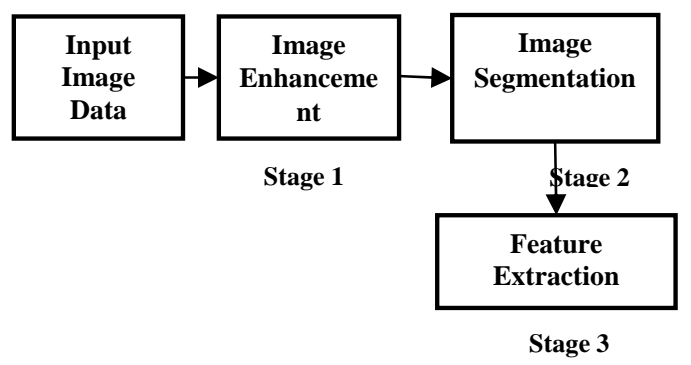

Figure 1: Three stage Breast Cancer Feature Extraction

Mammography is the most widespread technique used for diagnosing and detecting breast cancer. The intention of mammograms is a premature prediction of breast tumor, particularly through espial of masses and micro calcifications. Therapeutic specialists recite mammographic images and attempt to recognize the irregularities existing in the breast mass. It has been predicted that $10-30 \%$ of women interpret with breast cancer have false negative mammograms. Practically incorrect adverse mammograms are donated to doctor's catastrophe to recognize the breast tumor at premature phase due to deficiency of information or practice or misapprehension. Rendering to the therapeutic point of view, interpretation and clarification mammograms is actually a multifaceted process. CAD approaches are used to direct and swift up the investigative progression projected for numerous ailments such as analysis of numerous tumors cataloguing, wide-ranging of breast and lung tumor [3] and CAD paraphernalia customizes computer machineries to govern the anomalies contemporary in the mammogram such as masses, calcification through the assistance of thos consequences medical authorities can analyze and perceive breast tumor growth at initial phase [4]. The furthermost significant phase formerly employing two phases of CAD instrument is preprocessing phase, in which the segmentation of infected mass from other breast mass and the whole background is performed. The evaluations of mammogram images can assist radiologists in early diagnosis and detection of breast cancer tumor and diagnose the treatment very effectively [2]. As many methods had been used by various researchers for classifying malignant and benign masses, but only a few methods are capable of classifying breast mass region into malignant and benign masses. This can be attained by proposing some constraints as by using a number of different features.

In mammographic pre-processing, the contents of infected mass are identified by means of four echelons of gray level. Whereas relating the masses of mammographic images, those are encircled through pixels with small variations in gray levels and constitute plane borders [12]. Thus, that's very important to fastening the boundary depiction of the pixel weights for segmentation of breast masses. This tactic is directed on spatial province of image processing with the use of HSV color space possessions and that color space can be initially appropriate to human discernment colors and for every pixel a subjective value is determined using a gray weighted function which snatches that gray portion of the pixel and is actually vital in contradiction of noise and its parameters and given as [14]:

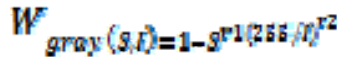

The $W_{\text {gray }}(S, I)$ is in between $(0-1)$ and is used to determine the gray part of pixel by means of intensity and it's saturated weights. For every pixel of concerned mass, a gray weighted value is determined and unwanted pixels are eliminated using thresholding approach as a pre-processing step. In gray thresholding, the average value of gray is determined as Gray Threshold $_{\text {and }}$ and values of pixel lower than Gray rhoshole are removed in the pre-processing step. To remove the blurring, which is introducing during the low pass filtering is removed by using high boost filter. Contrast, brightness and intensity problems are removed by using contrast stretching, histogram equalization, negativity and power law transformation etc [16].

\section{EXPERIMENTAL ANALYSIS}

Using modified thresholding, labeling algorithm and edge detection, segmentation of the mammogram image is carried out. Features such as geometric properties, textural properties and mathematical properties are calculated from the segmented image by their respective formula. These calculated features are used as an input to the MAS system. MAS system used these features for classifying the Breast cancer tumor ass malignant or benign. Present MAS system will not reduce the role of doctor/radiologist but it will provide a second opinion to the doctor. The result of this system along with doctor analysis will increase the accuracy of the diagnosis. Fig. 1 block diagram shows the overall process of the MAS system. Segmentation and feature extraction techniques are easily implemented and executed by using digital image processing toolbox of MATLAB software as shown in figure $2 \& 3$ and based on the results subjective analysis of both mammograms has been done.

The subjective observation is performed on the mammograms taking different samples of benign and malignant mammograms to observe the common distinctions between malignant and benign tumor mass. For that purpose, first the image is preprocessed using median and Weiner Filter. The median filter is a nonlinear digital filtering technique, often used to remove noise from an image or signal. Such noise reduction is a typical pre-processing step to improve the results of later processing (for example, edge detection on an image). Where, In signal processing, the Wiener filter is a filter used to produce an estimate of a desired or target random process by linear time-invariant (LTI) filtering of an 
observed noisy process, assuming known stationary signal and noise spectra, and additive noise.

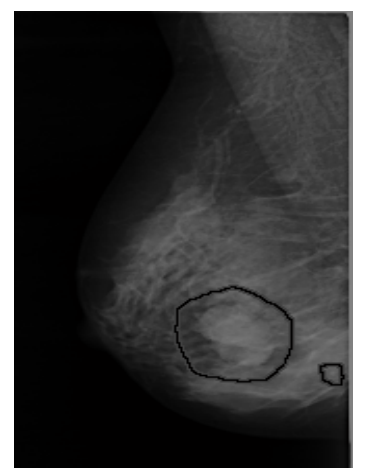

(a)Original Mammogram

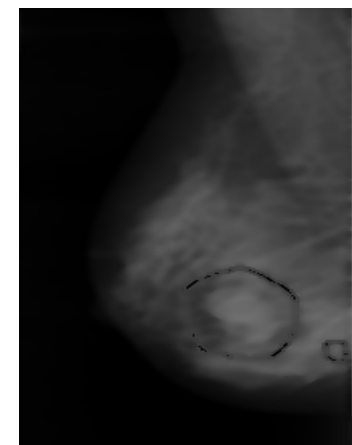
Removing Low Level Noises (c) Mammogram after

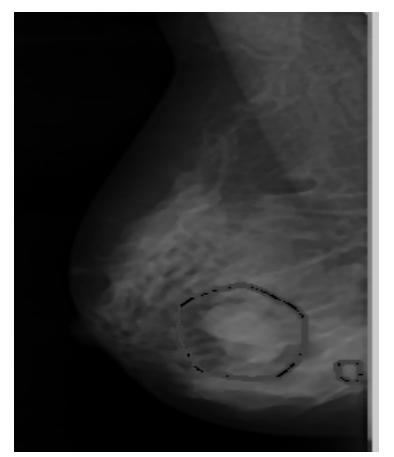
Low Level Noises

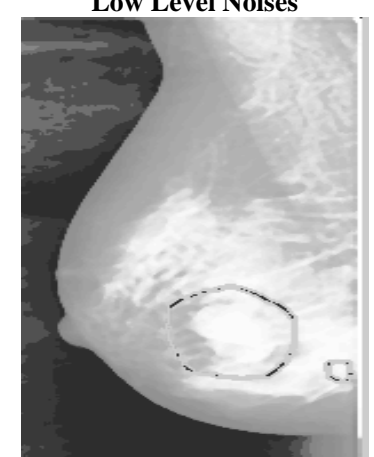

(d) Mammogram after Histogram Equalization (b) Mammogram after Removing

Figure 3: Benign Mammogram Pre-processing and it's Histogram Equalization

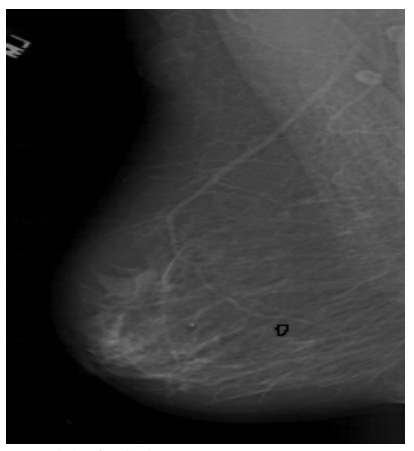

(a) Original Mammogram

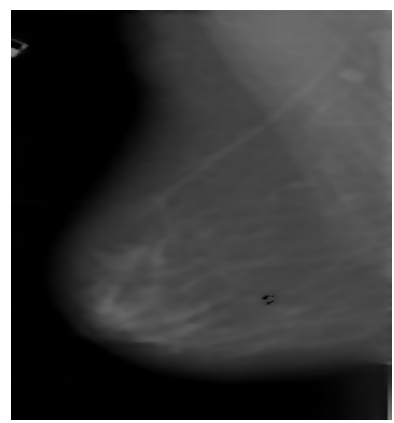

(c) Mammogram after Removing Low Level Noises

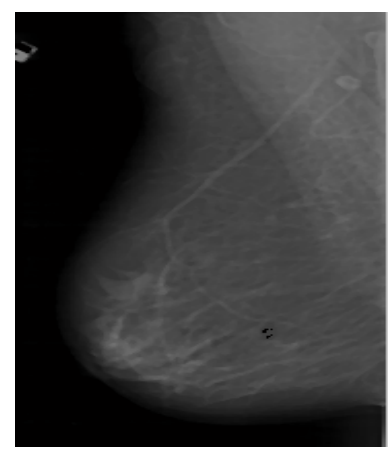

(b) Mammogram after Removing Low Level Noises

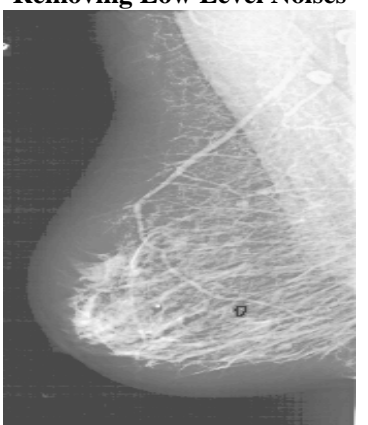

(d) Mammogram after Histogram Equalization
Figure 4: Malignant Mammogram Pre-processing and it’s Histogram Equalization

The Wiener filter minimizes the mean square error between the estimated random process and the desired process. The observed results are shown in in figure 3 and 4 . From figure $3,4(d)$, we can observe see that the contrast of the image has clearly been enhanced by performing equalization as compare the original mammogram images shown in figure 3,4 (a). In terms of qualitative judgment on the image, we can clearly see that the contrast is better because we are now able to see what was at the background and that the picture is really showing Objejects, which was not that obvious before.

From the subjective analysis of figure 3 (d), it is analyzed that, A benign tumor mass does not invade its surrounding tissue or spread around the body and their growth is slow. Further benign tumors tend to be very well-circumscribed, when looked at grossly, that is to say macroscopically, as shown in figure 3 . This means the edges of the benign tumor are usually very distinct and demarcated in a certain shape. From figure 4 (d), it is clear that malignant tumor mass is invade its surrounding tissue and spread around the body and malignant tumors grow more quickly in size. From that it is observed that, malignant tumors have an irregular shape and it may be difficult to tell where the tumor starts and ends.

\section{CONCLUSION}

Present MAS system will not reduce the role of doctor/radiologist but it will provide a second opinion to the doctor. The result of this system along with doctor analysis will increase the accuracy of the diagnosis. From the subjective analysis, it is concluded that, A benign tumor mass does not invade its surrounding tissue or spread around the body and their growth is slow with very wellcircumscribed, when looked at grossly, that is to say macroscopically. This means the edges of the benign tumor are usually very distinct and demarcated in a certain shape. It is also concluded from research that the malignant tumor mass is invade its surrounding tissue and spread around the body and malignant tumors grow more quickly in size. From that it is observed that, malignant tumors have an irregular shape and it may be difficult to tell where the tumor starts and ends.

\section{REFERENCES}

[1] “National Breast Cancer Organization”, http: //www.nationalbreastcancer.org/breast cancer.

[2] Muhic I., Williams C., "Fuzzy Analysis of Breast Cancer disease using Fuzzy-C-Means and Pattern Recognization”, Southeast Europe Journal of Soft Computing, vol. 4, pp. 50-55, 2014.

[3] Ohri, H Singh, A Sharma, "Classification of Breast Cancer Tumour using Statistical Features," International Journal for Science, Management and Technology (IJSMT) (7), 2016.

[4] Miranda G., Feleipe J., "Computer-aided diagnosis system based on fuzzy logic for breast Cancer categorization", International Journal of Computers in Biology and Medicine, vol.45, pp. 1008-1012, 2014.

[5] Jalalian A., Mashohor S., Karasfi B., "Computer aided diagnosis/detection in mammography and ultrasound: a review”, Elsevier Journal of Clinical Imaging, vol. 37, pp. 420426, 2012.

[6] Fuentes-Uriarte J., García M., Castillo O., “Comparative Study of Fuzzy Methods in Breast Cancer Diagnosis”, Proceedings of IEEE Conference, 2008.

[7] Walia N., Singh H., Tiwari S., Sharma A., "A Decision Support System for Tuberculosis Diagnosability,” International Journal on Soft Computing, vol. 6, pp 1-13,2015. 
[8] Nagarajasri B., Padmavathamma M., "Threshold Neuro Fuzzy Expert System for diagnosis of Breast Cancer,” International Journal of Computer Application, vol.66, pp 6-10, 2013.

[9] Caramahai M., Severin I., Balan H., "Evaluation of Breast Cancer Risk using Fuzzy Logic,” World Academy of Science and Technology, vol.56, pp37-42, 2009.

[10] American Cancer Society, "Learn about Breast Cancer”, http:// www.cancer.org,2011

[11] American College of Radiology, "Breast Imaging Reporting and Data System(BIRADS)", http:// www.acr.org,2010

[12] Shi X., Cheng H., "Detection and Classification of masses in breast ultrasound images," Proceedings of Elsevier Journal of Digital Image Processing, vol.20, pp. 824-836,2010

[13] Moon W., Chang J., Chen J., "Computer aided diagnosis of Breast masses using quantified BIRADS findings,”Proceedings of Elsevier Journal of Computer Methods and programs in Biomedicine, pp. 84-92,2013.

[14] Tang J., Ranyayyan R., "Computer Aided Detection and Diagnosis of Breast Cancer with Mammography: Recent Advances”, Proceedings of IEEE on Information Technology on Biomedical, vol. 2, pp 236-251, 2009

[15] for Screening Mammography (DDSM)", httpmarathon.csee.usf.edu/Mammography/Database.html

[16] Karabatak M., Ince M., “An Expert System for Diagnosis Breast Cancer based on Principal Component Analysis Method”, Proceedings of IEEE, vol.5,2008

[17] Keles A., Keles A., "Extracting Fuzzy Rules for Diagnosis of Breast Cancer”, Turkish Journal of Electrical Engineering and Computer Science, pp.1495-1503,2013. 